\title{
Soft-Pulse Dynamical Decoupling with Markovian Decoherence
}

\author{
Leonid P. Pryadko \\ Department of Physics \& Astronomy, University of California, Riverside, California, USA \\ Gregory Quiroz \\ Department of Physics, University of Southern California, Los Angeles, California, USA
}

\begin{abstract}
We consider the effect of broadband decoherence on the performance of refocusing sequences, having in mind applications of dynamical decoupling in concatenation with quantum error correcting codes as the first stage of coherence protection. Specifically, we construct cumulant expansions of effective decoherence operators for a qubit driven by a pulse of a generic symmetric shape, and for several sequences of $\pi$ - and $\pi / 2$-pulses. While, in general, the performance of soft pulses in decoupling sequences in the presence of Markovian decoherence is worse than that of the ideal $\delta$-pulses, it can be substantially improved by shaping.
\end{abstract}

\section{INTRODUCTION}

Dynamical decoupling [1, 2, 3] (DD) can be very effective in protecting coherence of a quantum system against low-frequency environment $4,5,6,7,8,9,10,11,12$, 13, 14, 15, 16, 17, 18, 19, 20, 21, 22, 23, 24, 25. This, combined with low resource requirement, makes it attractive as the first-level coherence protection technique, in combination with quantum error correcting codes 26, 27] (QECC). For such a combined decoherence protection technique to work universally, the performance of the DD should not be reduced in the presence of fast environment modes whose effect on coherence is to be dealt with by QECC.

Previously, the effects of broadband noise on DD were analyzed in a number of publications, with the primary target being the $1 / f$ or telegraph noise 16, 17, 28, 29, 30, 31. However, the effectiveness of refocusing sequences was mostly studied numerically, apart from special exactly-solvable cases 30, 32. No attempt has been made to investigate general properties and limitations of decoupling under these conditions. Certainly, there were no analytical studies of effects of pulse shaping on decoupling in the presence of broadband decoherence.

In this work, we concentrate on the dynamical decoupling (DD) of a single qubit (spin) in the presence of a broad-spectrum oscillator bath [Fig. 1]. We first consider the effect of the pulse shape in the special case where the external spin couplings can be described by a combination of a Markovian noise (described by the Lindblad decoherence operators) and a time-independent magnetic field. This basic problem is similar in importance to the canonical pulse-shaping for a nuclear spin in the presence of a chemical shift. For a qubit driven by an arbitrarily-shaped pulse, we construct an analytical expansion of the average decoherence operator, an analogue of the average Hamiltonian expansion 33, 34] but for the qubit density matrix evolution. Analyzing the first two terms of such expansions for several decoupling sequences, we formulate the conditions necessary for improved coherence of the qubit, and construct numerically the pulse shapes that satisfy these conditions. We then compare the performance of the obtained pulse shapes with "hard" pulses and the conventional first- and second-order nmr-style self-refocusing pulses in several decoupling sequences both analytically and numerically. For numerics, we model the oscillator bath as a combination of classical correlated gaussian noise and the Markovian noise [Fig. 1](a) and 11(c) respectively].

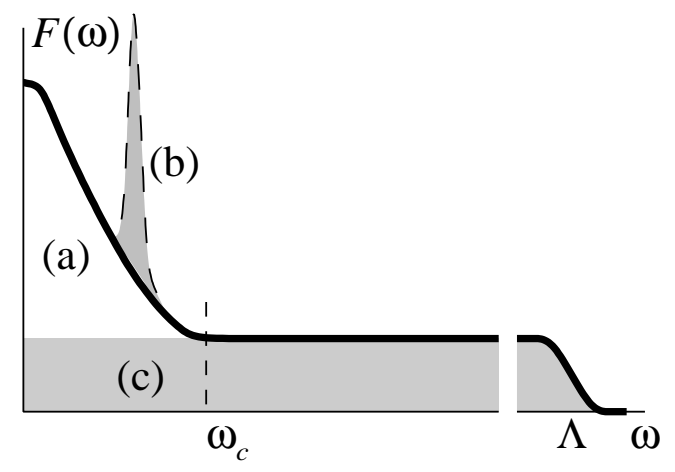

FIG. 1: Assumed spectral function of a generic oscillator bath (schematic). Dynamical decoupling can be very effective in preserving coherence in the presence of (a) featureless lowfrequency bath 21] and also (b) sharp resonances 35]. In this work we consider the effect of Markovian noise (c) on DD of a single qubit. We assume $F(\omega)=$ const up to a large cut-off frequency $\omega_{\max } \equiv \Lambda$, so that the qubit dynamics associated with the modes (c) can be modeled by the Linblad equation (11). When the high-frequency spectrum is not flat but is, e.g., a power law $\propto 1 / \omega^{\alpha}$ as in Refs. 30, 32, the decomposition into low-frequency (a) and Markovian (c) parts becomes approximate, with the Markovian decoherence rates $\tilde{\gamma}$ determined by $F(\omega)$ near $\omega=\Omega \equiv 2 \pi / \tau$ determined by the sequence duration $\tau$.

We analyze two kinds of decoupling sequences, the usual decoupling sequences utilizing $\pi$-pulses, and the sequences using composite pulses constructed of three $\pi / 2$ pulses similar to those used in the NMR WaHuHa 
experiment 33. While the former sequences interact relatively little with the decoherence operators $(\delta$-pulses leave the diagonal parts of the decoherence operators invariant), the latter ones are constructed to symmetrise the decoherence operator between all three channels. Such a redistribution seeks to minimize the detrimental effects of the non-Hermitian evolution caused by the decoherence operators on higher-level control sequences.

\section{MODEL}

\section{A. Hamiltonian}

A system of individually-controlled qubits in the presence of an oscillator thermal bath can be described by the following idealized Hamiltonian

$$
H=H_{C}(t)+H_{S}+H_{S B}+H_{B},
$$

where the control Hamiltonian

$$
H_{C}(t)=\frac{1}{2} \sum_{n, \alpha} V_{n}^{\alpha}(t) \sigma_{n}^{\alpha}
$$

the system Hamiltonian with one- and few-qubit terms,

$$
H_{S}=\frac{1}{2} \sum_{n, \alpha} B_{n}^{\alpha} \sigma_{n}^{\alpha}+\frac{1}{4} \sum_{n, n^{\prime}, \alpha, \beta} J_{n, n^{\prime}}^{\alpha \beta} \sigma_{n}^{\alpha} \sigma_{n^{\prime}}^{\beta}+\cdots
$$

the linear oscillator bath,

$$
H_{B}=\sum_{\mu} \omega_{\mu} a_{\mu}^{\dagger} a_{\mu}
$$

and the bath-coupling Hamiltonians,

$$
H_{S B}=\frac{1}{2} \sum_{n, \alpha} \hat{b}_{n}^{\alpha} \sigma_{n}^{\alpha}+\frac{1}{4} \sum_{n, n^{\prime}, \alpha, \beta} \hat{\mathrm{j}}_{n, n^{\prime}}^{\alpha \beta} \sigma_{n}^{\alpha} \sigma_{n^{\prime}}^{\beta}+\cdots
$$

Here $\sigma_{n}^{\alpha}, \alpha=x, y, z$, are the Pauli matrices for $n$-th qubit, $a_{\mu}, a_{\mu}^{\dagger}$ are the annihilation and creation operators for the oscillators of the bath, and the operators $\hat{b}_{n}^{\alpha}, \hat{\mathrm{j}}_{n n^{\prime}}^{\alpha \beta}$ describe the various couplings with the bath. A linear in phonons coupling, e.g., for the single-spin term can be written as

$$
\hat{b}_{n}^{\alpha}=\sum_{\mu} \frac{f_{n \mu}^{\alpha} a_{\mu}+\bar{f}_{n \mu}^{\alpha} a_{\mu}^{\dagger}}{\left(2 m_{\mu} \omega_{\mu}\right)^{1 / 2}}
$$

In the limit where the oscillator modes have a continuous spectrum, the kinetics of the decoherence effects is determined by the set of spectral coupling functions (matrices), e.g., for the single-qubit coupling,

$$
F_{n \alpha, n^{\prime} \beta}(\omega) \equiv \frac{\pi}{2} \sum_{\mu} \frac{f_{n \mu}^{\alpha} \bar{f}_{n^{\prime} \mu}^{\beta}}{m_{\mu} \omega_{\mu}} \delta\left(\omega-\omega_{\mu}\right)
$$

A schematic plot of a single representative component of this function is shown in Fig. 1.

For simplicity, we assume a combination of weak bath coupling and finite temperature $T$, so that the (generally non-Markovian) master equation 21, 36, 37, 38, 39] in the leading order ("Born approximation") is satisfied for the combination of $H_{S}+H_{C}(t)$. For the case of a single qubit, which is primarily discussed in this work,

$$
\begin{aligned}
\dot{\rho}= & -i\left[H_{S}+H_{C}(t), \rho\right]-\frac{1}{4} \int_{0}^{t} d t^{\prime} \mathcal{F}_{1}^{\alpha \beta}\left(t-t^{\prime}\right)\left[\sigma^{\alpha},\left[\sigma^{\beta}, \rho\right]\right] \\
& -\frac{i}{4} \int_{0}^{t} d t^{\prime} \mathcal{F}_{2}^{\alpha \beta}\left(t-t^{\prime}\right)\left[\sigma^{\alpha},\left\{\sigma^{\beta}, \rho\right\}\right],
\end{aligned}
$$

where the dissipation kernel $\hat{\mathcal{F}}_{1}(t)+i \hat{\mathcal{F}}(t) \equiv \hat{\mathcal{F}}(t)$ is defined as

$$
\hat{\mathcal{F}}(t)=\int_{0}^{\infty} \frac{d \omega}{\pi}\left[\hat{F}(\omega)\left(n_{\omega}+1\right) e^{i \omega t}+\hat{F}^{*}(\omega) n_{\omega} e^{-i \omega t}\right]
$$

$n_{\omega} \equiv \exp (\beta \omega)-1$ is the oscillator occupation number, and $\beta \equiv \hbar / T$.

Dynamical decoupling in the presence of a lowfrequency bath [Fig. 1 (a),(b)] was analayzed in Refs. 21, 35. General conclusion is that a carefully-designed sequence with the period $\tau \equiv 2 \pi / \Omega$ provides an excellent decoherence protection as long as the adiabaticity condition $\Omega \gtrsim \omega_{c}$ is satisfied. Here $\omega_{c}$ is the bath cut-off frequency such that all $\omega_{\mu}<\omega_{c}$ in Eq. (位).

More precisely, the analysis in Ref. 21 was done for a featureless low-frequency bath [Fig $1(\mathbf{a})]$ in the approximation of non-Markovian quantum kinetic (master) equation. The approach was to design a decoupling sequence effective for a closed system with the "frozen" bath, where each term in the bath-coupling Hamiltonian $H_{S B}[\mathrm{Eq}$. (5)] is replaced by the corresponding term with a non-zero $c$-number coefficient in Eq. (3) [e.g., $\hat{b}_{n}^{\alpha} \rightarrow B_{n}^{\alpha}$, $\hat{\mathrm{j}}_{n, n^{\prime}}^{\alpha, \beta} \rightarrow J_{n, n^{\prime}}^{\alpha, \beta}$, etc., with the matching indices.] The corresponding unitary evolution operator $U(t=\tau)$ after one decoupling period can be characterized by the decoupling order $K$, the number of terms in the Magnus (cumulant) expansion in powers of $H_{S}$ which are suppressed identically. Such a suppression can be expressed in terms of certain algebraic conditions which were used in the analysis of the solutions of the full master equation, orderby-order in powers of the adiabaticity parameter $\omega_{c} / \Omega$. At $K=1$, the single-phonon $T_{1}$ processes are completely suppressed as long as $\omega_{c}<\Omega$ (this can also be seen by analyzing the absorption spectra of the driven system 40 ). Additionally, since the bath coupling is modulated at the frequency $\Omega$, the effective dephasing rate is reduced by the factor of $\omega_{c} / \Omega 21$. At $K=2$, in many cases (e.g., for a single qubit), dephasing rate is suppressed faster than any power of $\omega_{c} / \Omega<1$ : terms of every order in the corresponding expansion are suppressed[21.

The description in terms of the master equation fails in the presence of sharp resonances [Fig. 1 $1(\mathbf{b})]$; the corresponding modes have to be included in the Hamil- 
tonian of the system and the controlled dynamics reanalyzed 35. Compared to qubits-only systems, presence of oscillators in $H_{S}$ can generate some additional terms in the Magnus expansion of the unitary evolution operator $U(\tau)$ for the closed system which requires more careful sequence design. When this is done, only the effective, DD-renormalized coupling to such modes matters. Nonequilibrium effects like mode heating do not occur as long as this renormalized coupling is small compared to either the width of the spectral peak or the corresponding frequency bias 35.

\section{B. Markovian decoherence}

We now consider the dynamical decoupling in the presence of broadband bath modes, see Fig. 11(c), assuming that the corresponding inverse correlation time $\Lambda \equiv \omega_{\max }$ is much larger than the control bandwidth which determines the pulse rate which can be implemented for the system. Out of the master equation (8), we separate a part $\mathcal{D}(\rho)$, Markovian on the time scale slow compared to $\omega_{\max }$, and assume that the remaining relatively slow modes are limited to frequencies $\omega \leq \omega_{c} \ll \omega_{\text {max }}$. Most generally, Markovian evolution implies the dissipator in the Lindblad form 41,

$$
\mathcal{D}(\rho) \equiv \frac{1}{2} \sum_{j}\left(\left[\Lambda_{j} \rho, \Lambda_{j}^{\dagger}\right]+\left[\Lambda_{j}, \rho \Lambda_{j}^{\dagger}\right]\right)
$$

where $\rho$ is the density matrix and $\Lambda_{j}$ are the decoherence operators. Such a form can also be recovered from Eq. (9) in the Markovian limit where the time-dependence of the kernel $\hat{\mathcal{F}}(t)$ becomes $\delta$-function-like. We note that this separation of the approximately Markovian part of the bath, Eq. (10), does not imply the consitions of high temperature or weak coupling combined with adiabaticity which are usually necessary for the applicability of the Markovian master equation [37, 39, 42].

For example, with flat part of $F(\omega) \approx$ const as in Fig. 1 (c), we could set the low-frequency cut-off $\omega_{c} \gtrsim \beta^{-1}$ and include the effect of $\left(2 n_{\omega}+1\right) \mathcal{F}_{1}(\omega)$ in Eq. (9) in the non-Markovian part of the bath kernel. This is precisely the case solved numerically in Sec. IV where the slow bath modes are replaced by correlated classical noise as appropriate for $\hbar \omega \lesssim T$.

To start, we will follow the approach of Ref. 21, and begin by analyzing the dynamics of the system with "frozen" dynamics of the slow modes in Fig. 1)(a). That is, we will assume that the decoupling frequency $\Omega$ is large compared to $\omega_{c}$, so that any bath couplings can be approximated by appropriate terms in $H_{S}$. Then, the effect of the fast environmental modes [Fig. 11(c)] on dynamical decoupling can be analyzed by considering the driven Lindblad equation,

$$
\dot{\rho}=-i\left[H_{C}(t)+H_{S}, \rho\right]+\mathcal{D}(\rho) .
$$

For a single qubit, parametrize the dissipator in terms of a Hermitian non-negative-definite rate matrix $\tilde{\gamma}_{\alpha \beta}$,

$$
\mathcal{D}(\rho)=\frac{1}{4} \sum_{\alpha, \beta=x, y, z} \tilde{\gamma}_{\alpha \beta}\left(\left[\sigma^{\alpha} \rho, \sigma^{\beta}\right]+\left[\sigma^{\alpha}, \rho \sigma^{\beta}\right]\right) .
$$

It is convenient to separate the symmetric real and antisymmetric imaginary parts of the rate matrix,

$$
\tilde{\gamma}_{\alpha \beta}=\gamma_{\alpha \beta}+i \epsilon_{\alpha \beta \gamma} \mathcal{R}_{\gamma}
$$

Then, if we rewrite the density matrix in terms of the Bloch vector $\mathbf{R} \equiv \mathbf{R}(t)$,

$$
\rho(t)=\frac{1}{2}(\mathbb{1}+\mathbf{R}(t) \cdot \vec{\sigma}), \quad R^{2} \leq 1,
$$

the equation for spin kinetics in the presence of the singlespin control (2) and system (3) Hamiltonians becomes

$$
\begin{aligned}
\dot{\mathbf{R}} & =[\mathbf{V}(t) \times \mathbf{R}]-\hat{\Gamma} \mathbf{R}-\overrightarrow{\mathcal{R}} \\
-\hat{\Gamma} \mathbf{R} & \equiv[\mathbf{B} \times \mathbf{R}]+(\hat{\gamma}-\mathbb{1} \operatorname{Tr} \hat{\gamma}) \mathbf{R}(t) .
\end{aligned}
$$

The terms with $\mathbf{V}(t)$ and $\mathbf{B}$ describe precession in the effective magnetic fields of the control and the system Hamiltonians, the term with $\hat{\gamma}$ describes the coherence loss due to Markovian bath, while the last term in Eq. (15) ensures the correct equilibrium value of $\mathbf{R}$ in the absence of control.

If we work in the basis associated with the dissipator, the rate matrix $\hat{\gamma}$ is diagonal. In particular, for the case of nuclear magnetic resonance (or any physical qubit with large energy difference between the levels), we have $\gamma_{x x}=$ $\gamma_{y y}=\gamma, \gamma_{z z}=\gamma_{\phi}$, and the matrix (16) takes the form

$$
\hat{\Gamma}=\left(\begin{array}{ccc}
\gamma+\gamma_{\phi} & B_{z} & -B_{y} \\
-B_{z} & \gamma+\gamma_{\phi} & B_{x} \\
B_{y} & -B_{x} & 2 \gamma
\end{array}\right)
$$

These decay rates correspond to the usual coherence times $T_{1}^{-1}=2 \gamma, T_{2}^{-1}=\gamma+\gamma_{\phi}$. The corresponding vector $\mathcal{R}$ has only one component, $\mathcal{R}_{z} \geq 0$. In the special case $\gamma_{\phi}=\gamma$, the rate matrix is proportional to the identity matrix, $\hat{\gamma}=\gamma \mathbb{1}$.

We emphasize again that, while the analytical analysis is done assuming time-independent "magnetic field" $\mathbf{B}$ in Eq. (17), the decoupling accuracy for obtained pulse shapes is also verified numerically in Sec. [IV] with the components $B_{\mu}(t)$ taken as a classical time-dependent Gaussian correlated noise.

\section{Interaction representation \& Magnus expansion}

In dynamical decoupling, it is the control Hamiltonian $H_{C}(t)$ that dominates the dynamics. The evolution associated with the system Hamiltonian $H_{S}$ [Eq. (3)] or the dissipator $\mathcal{D}(\rho)$ [Eq. (10)] is much slower. Thus, it is convenient to consider the dynamics in the interaction 
representation with respect to $H_{C}(t)$. To this end, we introduce the non-perturbed unitary evolution operator $U_{0} \equiv U_{0}(t)$,

$$
U_{0}(t)=T_{t} \exp \left(-i \int_{0}^{t} d t^{\prime} H_{C}\left(t^{\prime}\right)\right)
$$

where $T_{t}$ represents the usual time-ordering operator, and the corresponding orthogonal spin-rotation matrix, $\hat{Q}_{0} \equiv \hat{Q}_{0}(t)$, can be computed as follows

$$
U_{0} \sigma^{\alpha} U_{0}^{\dagger}=Q_{0}^{\alpha \beta} \sigma^{\beta}
$$

The components $Q_{0}^{\alpha \beta}$ satisfy the Bloch equation

$$
\dot{Q}_{0}^{\alpha \beta}(t)=e^{\alpha \gamma \delta} V^{\gamma}(t) Q_{0}^{\delta \beta}(t), \quad \hat{Q}_{0}(0)=\mathbb{1} .
$$

If we write the solution of the uniform version of Eq. (15) (i.e., with $\overrightarrow{\mathcal{R}}=0$ ) in terms of the full evolution matrix $\hat{Q} \equiv \hat{Q}(t)$,

$$
\mathbf{R}(t)=\hat{Q}(t) \mathbf{R}_{0}
$$

we can introduce the decomposition $\hat{Q}=\hat{Q}_{0} S$, where the matrix $S$ defines the slow evolution of the Bloch vector in the rotating frame defined by the control fields. The equation for $S$ reads

$$
\dot{S}=-\hat{\Gamma}(t) S, \quad \hat{\Gamma}(t) \equiv \hat{Q}_{0}^{\mathrm{t}}(t) \hat{\Gamma} \hat{Q}_{0}(t),
$$

where $\hat{\Gamma}(t)$ is the evolution operator 16 in the interaction representation. The formal solution of Eq. (22) can be again written as a time-ordered exponent,

$$
S(t)=T_{t} \exp \left(-\int_{0}^{t} d t^{\prime} \hat{\Gamma}\left(t^{\prime}\right)\right)
$$

For a periodic control field, $H_{c}(t+\tau)=H_{c}(t)$, such that the zeroth-order rotation matrix is also periodic, $Q_{0}(t+\tau)=Q_{0}(t)$, the time-ordered exponent can be evaluated in terms of the average decoherence operator $\bar{\Gamma}$, an analogue of the average Hamiltonian [33, 34]:

$$
S(n \tau)=e^{-n \tau \bar{\Gamma}}, \quad \bar{\Gamma} \equiv \bar{\Gamma}^{(0)}+\bar{\Gamma}^{(1)}+\cdots,
$$

where

$$
\begin{aligned}
& \bar{\Gamma}^{(0)}=\frac{1}{\tau} \int_{0}^{\tau} d t_{1} \tilde{\Gamma}\left(t_{1}\right) \\
& \bar{\Gamma}^{(1)}=-\frac{1}{2 \tau} \int_{0}^{\tau} d t_{2} \int_{0}^{t_{2}} d t_{1}\left[\hat{\Gamma}\left(t_{2}\right), \hat{\Gamma}\left(t_{1}\right)\right]
\end{aligned}
$$

etc. Generally, the term $\bar{\Gamma}^{(k)}, k>0$, of this expansion contains a $(k+1)$-fold integration of commutators of the rotating-frame decoherence operator $\hat{\Gamma}\left(t_{j}\right)$; it scales as $\propto \tau^{k}$. Trace of a commutator is zero, thus $\operatorname{Tr} \bar{\Gamma}^{(k)}=0$ for all $k>0$; this implies

$$
\operatorname{Tr} \bar{\Gamma}=\operatorname{Tr} \bar{\Gamma}^{(0)}=\operatorname{Tr} \hat{\Gamma}=2 \operatorname{Tr} \hat{\gamma}
$$

Generally, the trace-conserving modifications of the effective decoherence operator correspond to (some degree of) symmetrization between the decoherence channels. More precisely, the real part of the smallest eigenvalue of $\bar{\Gamma}$ cannot be smaller than that of $\hat{\Gamma}$. This can be seen by separating out the trivial part of $\hat{\Gamma}$ proportional to identity matrix, which corresponds to uniform decoherence for all channels and remains unchanged in the interaction representation 22 .

\section{Coherence loss in controlled system}

As would be expected on general grounds, Eq. (27) implies that the DD cannot eliminate the Markovian dissipation; at best we can hope to eliminate the off-diagonal terms of matrix $\hat{\Gamma}$ and redistribute the rates over the decoherence channels. For example, for an NMR qubit decoherence model, Eq. (17), redistribution (symmetrization) over all three channels would lead to

$$
\bar{\Gamma}=\frac{2}{3}\left(2 \gamma+\gamma_{\phi}\right) \mathbb{1}
$$

With $\gamma \ll \gamma_{\phi}$, this is equivalent to a $33 \%$ reduction of the maximum decoherence rate, or a $50 \%$ increase of the coherence time measured by the fidelity minimized over the initial conditions.

We should note that the quoted estimate for the maximum decoherence rate improvement does not take into account the antisymmetric part of the rate matrix $\tilde{\gamma}$ [Eq. (13)], or, equivalently, the non-uniform term $\overrightarrow{\mathcal{R}}$ in Eq. (15). In the absence of control fields, this term is responsible for asymptotic thermal-equilibrium orientation of the spin; it is small and can be ignored at sufficiently high temperatures (e.g., room-temperature NMR), but it can be large at temperatures small compared to the qubit level difference $\Delta E_{01}=|\mathbf{B}|$.

We can drop the non-uniform term $\overrightarrow{\mathcal{R}}$ in the analysis of decoherence since it does not affect the evolution of the average fidelity, that is, the fidelity averaged by the initial conditions, $\overline{\mathcal{F}}(t) \equiv \operatorname{Tr}\langle\rho(0) \rho(t)\rangle_{0}$. Indeed, Eq. (15) is a set of linear differential equations for the components of the Bloch vector $\mathbf{R}(t)$; the solution with given initial conditions $\mathbf{R}(0) \equiv \mathbf{R}_{0}$ can be written as a sum of the solution (21) of the uniform equation with the same initial condition, and that of the non-uniform equation but with zero initial condition. In the expression for the average fidelity,

$$
\overline{\mathcal{F}}(t)=\frac{1}{2}\left(\mathbb{1}+\left\langle\mathbf{R}_{0} \cdot \mathbf{R}(t)\right\rangle_{\mathbf{R}_{0}}\right)
$$

the solution of the uniform equation is bilinear in the components of $\mathbf{R}_{0}$ while the non-uniform part is linear; the averaging over all directions of $\mathbf{R}$ leaves only the bilinear part,

$$
\overline{\mathcal{F}}(n \tau)=\frac{1}{2}+\frac{1}{6} \operatorname{Tr} \hat{Q}(n \tau)=\frac{1}{2}+\frac{1}{6} \operatorname{Tr} e^{-n \tau \bar{\Gamma}},
$$


where we assumed the zeroth-order rotation matrix to be periodic, $\hat{Q}_{0}(n \tau)=\mathbb{1}$, and used the representation (24) in terms of the average decoherence operator $\bar{\Gamma}$.

The obtained expression (30), along with the properties of the average decoherence operator $\bar{\Gamma}$ discussed in the previous section [see Eq. (27)], imply that the average fidelity cannot be improved by means of dynamical decoupling beyond eliminating the effect of the system Hamiltonian $H_{S}$ [Eq. (3)]. With $\mathbf{B}=0$, this follows directly from the convexity of exponentials in Eq. (30) if one works in the basis where the average decoherence matrix $\bar{\gamma}=\operatorname{diag}\left(\gamma_{1}, \gamma_{2}, \gamma_{3}\right), 0 \leq \gamma_{1} \leq \gamma_{2} \leq \gamma_{3}$. In particular, the maximum possible fidelity with given $\operatorname{Tr} \hat{\gamma}$ corresponds to only one non-zero component, $\gamma_{3}=\operatorname{Tr} \hat{\gamma}$. In this case two (or any even number) qubit errors compensate each other. On the other hand, the symmetric case $\gamma_{1}=\gamma_{2}=\gamma_{3}=\operatorname{Tr} \hat{\gamma} / 3$ in the absence of error correction corresponds to the fidelity minimum.

We should also note that a symmetrization of decoherence rates over channels [complete as in Eq. (28), or partial] would prohibit the use of special QECCs designed for strongly asymmetric error rates between the channels 43, 44, 45, 46, 47, 48.

In this work we assume that the benefits of dynamical decoupling (due to reduced decoherence coming from the slow degrees of freedom) outweigh the detrimental effects related to symmetrization of the coherence rates.

\section{PULSE AND SEQUENCE CHARACTERIZATION}

\section{A. Hard pulses}

Let us now use the obtained formalism to analyze controlled dynamics. We start with hard pulses, with the $\delta$-function centered at the middle of the interval of duration $\tau_{p}$. For example, for a $\left(\phi_{0}\right)_{x}$ pulse (rotation angle $\phi_{0}$ with respect to $x$-axis), the non-zero control field in Eq. (2) can be written as

$$
V^{x}(t)=\phi_{0} \delta\left(t-\tau_{p} / 2\right), 0<t<\tau_{p}
$$

For the special case $\phi_{0}=\pi$ (pulse $\pi_{x}$ ), the corresponding rotation matrix (20) is

$$
\hat{Q}_{0}(t)=\left\{\begin{array}{cc}
\mathbb{1}, & 0<t<\tau_{p} / 2 \\
\operatorname{diag}(1,-1,-1), & \tau_{p} / 2<t<\tau_{p}
\end{array}\right.
$$

Over the second half of the interval, the components $\Gamma_{x y}$ and $\Gamma_{x z}$ are inverted; as a result in the leading order

$$
\bar{\Gamma}^{(0)}\left(\pi_{x}\right)=\left(\begin{array}{ccc}
\gamma_{y y}+\gamma_{z z} & & \\
& \gamma_{z z}+\gamma_{x x} & B_{x}-\gamma_{y z} \\
& -B_{x}-\gamma_{y z} & \gamma_{x x}+\gamma_{y y}
\end{array}\right)
$$

The expression for $\bar{\Gamma}^{(1)}\left(\pi_{x}\right)$ is too complicated to quote here. However, for the symmetric sequence $2 \mathrm{~s} \equiv \mathrm{XX}$ of
$X \equiv \pi_{x}$ pulses (which is the repeated part of the CarrPurcell sequence), the first-order average decoherence operator $\bar{\Gamma}^{(1)}(\mathbf{2 s})=0$, while the zeroth-order term $\bar{\Gamma}^{(0)}(\mathbf{2 s})$ is the same as for a single pulse given by Eq. (33).

Deriving similar expressions for a $\pi_{y}$ pulse, we can easily construct the expressions for standard twodimensional decoupling sequences of hard $\pi$-pulses. In particular, the group-averaging 10, 49] 4-pulse sequence $\mathbf{4 p} \equiv \mathrm{XY} \overline{\mathrm{X} Y}$ results in cancellation of all off-diagonal terms to leading order,

$$
\bar{\Gamma}^{(0)}(\mathbf{4 p})=\left(\begin{array}{ccc}
\gamma_{y y}+\gamma_{z z} & 0 & 0 \\
0 & \gamma_{x x}+\gamma_{z z} & 0 \\
0 & 0 & \gamma_{x x}+\gamma_{y y}
\end{array}\right)
$$

while the symmetric sequence $\mathbf{8 s} \equiv \mathrm{XY} \overline{\mathrm{X}} \mathrm{YY} \overline{\mathrm{X}} \mathrm{YX}$ (symmetrized version of $\mathbf{4 p}$ ) results in cancellation of all offdiagonal terms $\left[\bar{\Gamma}^{(0)}(\mathbf{8 s})=\bar{\Gamma}^{(0)}(\mathbf{4} \mathbf{p})\right.$, see Eq. (34) $]$ and does not produce any 1st-order corrections, $\bar{\Gamma}^{(1)}(\mathbf{8 s})=0$. We note that with the hard pulses, the contribution to $\bar{\Gamma}^{(2)}(\mathbf{8 s})$ is non-zero already for a Hamiltonian evolution with $\hat{\gamma}=0$, as long as both $B_{x}$ and $B_{y}$ are non-zero.

The hard $\pi$ pulses do not modify the structure of the diagonal part of the evolution matrix $\Gamma$. A redistribution (symmetrization) over different decoherence channels can be done if we deliberately orient the spin along different axes with $\pi / 2$-pulses. In the absence of the system Hamiltonian $(\mathbf{B}=0)$, for NMR decoherence model (17), the symmetrization can be achieved with the WaHuHa (MREV-4) NMR experiment [33, 50], a sequence of $\pi / 2$ pulses with the cycle $\mathbf{5}=\mathrm{XY} 0 \mathrm{YX}$ of duration $\tau=5 \tau_{p}$, where each pulse is centered as in Eq. (31) and 0 stands for a free evolution interval equal to the pulse duration $\tau_{p}$. The averaging of the second-rank coupling achieved by this sequence is used to eliminate dipolar coupling between nuclei. However, the accuracy achieved by this sequence is rather sensitive to chemical shifts; the corrections are present already in the leading order effective evolution operator.

To achieve both the decoherence symmetrization and the decoupling, we constructed several pulse sequences based upon the composite pulses $R_{\alpha} \equiv \mathrm{X} \bar{Y} \mathrm{X}, R_{\beta} \equiv$ $\mathrm{XYX}$, and the inverted versions $\bar{R}_{\alpha} \equiv \overline{\mathrm{X}} \mathrm{Y} \overline{\mathrm{X}}$, and $\bar{R}_{\beta} \equiv$ $\overline{\mathrm{X}} \overline{\mathrm{Y}} \overline{\mathrm{X}}$. Here, $X$ and $Y$ now denote the $\pi / 2$ pulses applied in the corresponding directions. The composite pulses $R_{\alpha}$ and $R_{\beta}$ are mutually orthogonal $\pi$-pulses constructed in such a way that the spin's $x, y$, and $z$ axes spend equal time oriented along the $z$-axis. As a result, they achieve a leading-order symmetrization of the NMR decohernce matrix, see Eq. (17) with $\vec{B}=0$. The twelveand twenty-four pulse sequences, 12 and 24 respectively, are merely the universal decoupling sequences $4 \mathbf{p}$ and $\mathbf{8 s}$ constructed from these composite pulses,

$$
\begin{aligned}
& \mathbf{1 2}=R_{\alpha} R_{\beta} \bar{R}_{\alpha} R_{\beta}, \\
& \mathbf{2 4}=R_{\alpha} R_{\beta} \bar{R}_{\alpha} R_{\beta} R_{\beta} \bar{R}_{\alpha} R_{\beta} R_{\alpha},
\end{aligned}
$$

while the 48-pulse sequence includes an additional cycle of phase ramping analogous to that in MLEV-16 and 
higher order sequences 51,

$$
\mathbf{4 8}=R_{\alpha} R_{\beta} \bar{R}_{\alpha} R_{\beta} \bar{R}_{\alpha} \bar{R}_{\beta} R_{\alpha} \bar{R}_{\beta}+\text { (reverse). }
$$

The basic 12-pulse sequence achieves symmetrization but not the decoupling; the leading-order average decoherence operator reads

$$
\bar{\Gamma}_{\mathbf{1 2}}^{(0)}=\frac{1}{6}\left(\begin{array}{ccc}
4\left(2 \gamma+\gamma_{\phi}\right) & 2 B_{y} & -B_{z} \\
-2 B_{y} & 4\left(2 \gamma+\gamma_{\phi}\right) & -B_{z} \\
B_{z} & B_{z} & 4\left(2 \gamma+\gamma_{\phi}\right)
\end{array}\right)
$$

The longer sequence $\mathbf{2 4}$ achieves both the decoupling and symmetrization of the decoherence operator (17) in the leading order, i.e., $\bar{\Gamma}_{\mathbf{2 4}}^{(0)}$ is given by Eq. (28). The sequence 48 achieves symmetrization to subleading order, i.e., $\bar{\Gamma}_{\mathbf{4 8}}^{(0)}$ is given by Eq. (28) while $\bar{\Gamma}_{48}^{(1)}=0$. Moreover, in the experimentally relevant case $B_{x}=B_{y}=0$, the $\bar{\Gamma}_{\mathbf{4 8}}^{(2)}$ only has non-zero elements $\propto B_{z}^{2}\left(\gamma-\gamma_{\phi}\right) \tau_{p}^{2}$ along the diagonal.

\section{B. Finite-duration pulses}

Let us now analyze the performance of discussed sequences when finite-duration "soft" pulses are used instead of the ideal hard pulses. Basically, we extend the formalism of Refs. 35, 52 to the case of non-Hermitian evolution (15) with the most general symmetric rate matrix $\hat{\gamma}$.

\section{Average evolution operator for a single pulse}

We begin with the qubit evolution driven by a onedimensional pulse of arbitrary shape,

$$
H_{c}(t)=\frac{1}{2} V(t) \sigma^{x} .
$$

The (zeroth-order) evolution operator due to control field alone is given by

$$
U_{c}(t)=\exp \left(-i \sigma^{x} \phi(t) / 2\right), \quad \phi(t)=\int_{0}^{t} d t^{\prime} V\left(t^{\prime}\right) .
$$

This is just a rotation by angle $\phi(t)$ around the $x$-axis,

$$
\hat{Q}_{0}(t)=\left(\begin{array}{ccc}
1 & 0 & 0 \\
0 & \cos \phi(t) & \sin \phi(t) \\
0 & -\sin \phi(t) & \cos \phi(t)
\end{array}\right) .
$$

With non-zero decoherence matrix $\hat{\gamma} \neq 0$, the evolution operator in the interaction representation, Eq. (22), will contain terms that depend both linearly and bi-linearly on the components of the matrix (41). In other words, the time-dependence of $\hat{\Gamma}(t)$ will be only through terms proportional to $\cos \phi, \sin \phi, \cos 2 \phi$, and $\sin 2 \phi$. The functions of the doubled angle are specifically due to the Markovian decoherence operators; they were not present in the analysis of Hamiltonian dynamics in Refs. 35,52 .

For the symmetric pulse shape,

$$
V_{x}\left(\tau_{p}-t\right)=V_{x}(t), \quad \phi\left(\tau_{p}-t\right)=\phi_{0}-\phi(t),
$$

it is convenient to introduce the symmetrized angle

$$
\varphi(t) \equiv \phi(t)-\phi_{0} / 2, \quad \varphi\left(\tau_{p}-t\right)=-\varphi(t) .
$$

The averages of time-dependent terms can be then written as follows:

$$
\begin{aligned}
& \langle\cos \phi\rangle=v \cos \frac{\phi_{0}}{2}, \quad\langle\sin \phi\rangle=v \sin \frac{\phi_{0}}{2}, \\
& \langle\cos 2 \phi\rangle=v_{2} \cos \phi_{0}, \quad\langle\sin 2 \phi\rangle=v_{2} \sin \phi_{0},
\end{aligned}
$$

where

$$
v \equiv\langle\cos \varphi(t)\rangle, \quad v_{2} \equiv\langle\cos 2 \varphi(t)\rangle,
$$

and the pulse-averages are defined simply as

$$
\langle f(t)\rangle \equiv \frac{1}{\tau_{p}} \int_{0}^{\tau_{p}} d t f(t)
$$

We see that, to leading order, the pulse-shape dependence of the average evolution operator $\bar{\Gamma}^{(0)}$ [Eq. (25)] is reduced to only two constants. The parameter $v$ gives an effective pulse length to first order; it is equal to zero, $v=0$ for ideal $\delta$-inversion pulses (rotation angle $\phi_{0}=\pi$ ), as well as for Hermitian pulses 53. or other 1st-order self-refocusing pulses 19, 52, 54. The parameter $v_{2}$ vanishes for ideal $\delta$-pulses with the rotation angle $\phi_{0}=\pi / 2$; for hard inversion pulses [Eq. (31)] we have $\varphi= \pm \pi / 2$, thus $\cos 2 \varphi=-1$ throughout the interval, which gives $v_{2}=-1$. For soft pulses with limited amplitude, $\left|v_{2}\right|<1$. The values of the parameters for some other pulse shapes are listed in Table [.

Specifically for a symmetric $\pi_{x}$ pulse, we get

$$
\bar{\Gamma}^{(0)}\left(\pi_{x}\right)=\left(\begin{array}{ccc}
\gamma_{y y}+\gamma_{z z} & v\left(B_{y}+\gamma_{x z}\right) & v\left(B_{z}-\gamma_{x y}\right) \\
v\left(-B_{y}+\gamma_{x z}\right) & \gamma_{x x}+\gamma_{y y} \frac{1+v_{2}}{2}+\gamma_{z z} \frac{1-v_{2}}{2} & v_{2} \gamma_{y z}+B_{x} \\
-v\left(B_{z}+\gamma_{x y}\right) & v_{2} \gamma_{y z}-B_{x} & \gamma_{x x}+\gamma_{y y} \frac{1-v_{2}}{2}+\gamma_{z z} \frac{1+v_{2}}{2}
\end{array}\right),
$$

which goes over to Eq. (33) for $v=0, v_{2}=-1$. 
The first-order average evolution operator, Eq. 26, contains the double integral of the commutator of the rotating-frame evolution operator $\hat{\Gamma}\left(t_{i}\right)$ taken at different moments $t_{i}, i=1,2$. There are only a few combinations of trigonometric functions of the symmetrized angle (43) and the time-independent terms that result in non-zero contributions to $\bar{\Gamma}^{(1)}$ for a single symmetric pulse with the duration $\tau_{p}$ :

$$
\begin{aligned}
\alpha & \equiv\left\langle\sin \left(\varphi-\varphi^{\prime}\right)\right\rangle, \\
\zeta & \equiv\left\langle\left(\frac{t}{\tau_{p}}-\frac{1}{2}\right) \sin \varphi\right\rangle, \\
\alpha_{2} & \equiv\left\langle\sin \left(2 \varphi-2 \varphi^{\prime}\right)\right\rangle, \\
\zeta_{2} & \equiv\left\langle\left(\frac{t}{\tau_{p}}-\frac{1}{2}\right) \sin 2 \varphi\right\rangle, \\
\mu & \equiv\left\langle\sin \left(2 \varphi-\varphi^{\prime}\right)\right\rangle,
\end{aligned}
$$

where $\varphi \equiv \varphi(t), \varphi^{\prime} \equiv \varphi\left(t^{\prime}\right)$, and the two-parameter averages are defined as

$$
\left\langle f\left(t, t^{\prime}\right)\right\rangle \equiv \frac{1}{\tau_{p}^{2}} \int_{0}^{\tau_{p}} d t \int_{0}^{t} d t^{\prime} f\left(t, t^{\prime}\right)
$$

The parameters $\alpha$ and $\zeta$ completely characterize the Hermitian evolution during the pulse; they were introduced and discussed in detail in Refs. $35,52$.

\section{Decoupling sequences of finite-length $\pi$-pulses}

Given the computed terms of the average evolution operator for a single finite-duration pulse along the $x$ axis, the corresponding expressions for a pulse along an arbitrary direction can be found by an orthogonal transformation, using the appropriately transformed vector
$\mathbf{B}$ and decoherence matrix $\hat{\gamma}$. We checked that in simple cases, $\hat{\mathbf{n}}= \pm \mathbf{e}_{x}$ and $\hat{\mathbf{n}}= \pm \mathbf{e}_{y}$, the results coinside identically with those obtained directly as outlined in Sec. III B 1.

With these expressions, the evolution matrix $\hat{Q}$ for a sequence of pulses can be computed as a product of the evolution operators for individual pulses,

$$
\hat{Q}_{i}=\hat{Q}_{0, i}\left[\mathbb{1}-\tau_{p} \bar{\Gamma}_{i}^{(0)}-\tau_{p} \bar{\Gamma}_{i}^{(1)}+\frac{\tau_{p}^{2}}{2} \bar{\Gamma}_{i}^{(0)} \cdot \bar{\Gamma}_{i}^{(0)}+\mathcal{O}\left(\tau_{p}^{3}\right)\right]
$$

For a sequence of pulses, the net average evolution operator $\bar{\Gamma}_{\text {seq }}$ can be obtained in terms of a logarithm of the obtained series (multiplied from the left by the corresponding $\hat{Q}_{0 \text {,seq }}$ when it is not equal to $\mathbb{1}$ ).

For the cycle $\mathbf{2 s}=\mathrm{XX}$ of the Carr-Purcell sequence, the leading-order evolution operator is the same as for a single $\pi_{x}$-pulse, Eq. (48), but with $v \rightarrow 0$. The corresponding expression in the subleading order is complicated and contains terms proportional to $v, v^{2}, v v_{2}, \alpha$, $\alpha_{2}, \zeta_{2}$; all of these terms disappear in the limit of hard pulses. An antisymmetric version of the same sequence, $\mathbf{2} \mathbf{a}=\overline{\mathrm{X} X}$, results in the leading-order evolution operator of the form (48), including the terms with $v$, but the subleading order disappears, $\bar{\Gamma}^{(1)}(\mathbf{2 a})=0$. Finally, for the phase-ramped four-pulse sequence $\mathbf{4 a}=\overline{\mathrm{X}} \overline{\mathrm{X}} \mathrm{XX}$, the leading-order effective decoherence operator is the same as for the Carr-Purcell cycle,

$$
\bar{\Gamma}^{(0)}(\mathbf{4 a})=\bar{\Gamma}^{(0)}(\mathbf{2} \mathbf{s})=\left.\bar{\Gamma}^{(0)}(\mathrm{X})\right|_{v \rightarrow 0},
$$

while the subleading order vanishes, $\bar{\Gamma}^{(1)}(\mathbf{4 a})=0$.

For the two-dimensional decoupling sequence $\mathbf{4 p}=$ $\mathrm{XY} \overline{\mathrm{X}} \mathrm{Y}$, the leading-order effective decoherence operator reads

$$
\bar{\Gamma}^{(0)}(\mathbf{4} \mathbf{p})=\left(\begin{array}{ccc}
\gamma_{y y}+\gamma_{z z} \frac{3-v_{2}}{4}+\gamma_{x x} \frac{1+v_{2}}{4} & -\frac{v}{2}\left(B_{x}+\gamma_{y z}\right) & \frac{v}{2}\left(\gamma_{x y}-B_{z}\right) \\
\frac{v}{2}\left(B_{x}-\gamma_{y z}\right) & \gamma_{x x}+\gamma_{z z} \frac{3-v_{2}}{4}+\gamma_{y y} \frac{1+v_{2}}{4} & 0 \\
\frac{v}{2}\left(B_{z}+\gamma_{x y}\right) & 0 & \left(\gamma_{x x}+\gamma_{y y}\right) \frac{3-v_{2}}{4}+\gamma_{z z} \frac{1+v_{2}}{2}
\end{array}\right)
$$

Note that the corresponding diagonal terms are determined by the diagonal terms of the original matrix $\hat{\gamma}$ and the parameter $v_{2}$ [see Eq. (46)], while off-diagonal terms are proportional to the effective width of the pulse $v$. For Hamiltonian evolution, $\hat{\gamma}=0$, one could achieve $\bar{\Gamma}^{(0)}(\mathbf{4} \mathbf{p})=0$ by using self-refocusing pulses with $v=0$. The next order term is complicated, but it is eliminated for the antisymmetric sequence, 8a $=\mathrm{XY} \overline{\mathrm{X}} \mathrm{Y} \overline{\mathrm{Y}} \mathrm{X} \overline{\mathrm{X}}$. Specifically, for this sequence we obtain the same leadingorder expression $\bar{\Gamma}^{(0)}(\mathbf{8 a})=\bar{\Gamma}^{(0)}(\mathbf{4} \mathbf{p})$ [Eq. (55)], and
$\bar{\Gamma}^{(1)}(\mathbf{8} \mathbf{a})=0$. On the other hand, for the symmetric sequence $\mathbf{8 s}=\mathrm{XY} \overline{\mathrm{X}} Y \mathrm{YX} Y \mathrm{X}$, it is the leading-order offdiagonal corrections proportional to $v$ that vanish,

$$
\bar{\Gamma}^{(0)}(\mathbf{8 s})=\left.\bar{\Gamma}^{(0)}(\mathbf{4} \mathbf{p})\right|_{v \rightarrow 0}
$$

while the subleading order contains terms proportional to $v, \alpha, \alpha_{2}$, and $\zeta_{2}$. All of the corrections can be eliminated by a supercycle of phase ramping which leads to an antisymmetric 16-pulse sequence $\mathbf{1 6 a}$ constructed as the $\mathbf{8 s}$ sequence followed by the same sequence with inverted 


\begin{tabular}{|c|c|c|c|c|c|c|c|c|}
\hline pusise & $\varphi_{0}$ & - & $U_{2}$ & $\alpha / 2$ & $\alpha_{2} / 2$ & 3 & $\zeta_{2}$ & $\mu$ \\
\hline$\overline{\phi_{0} \delta\left(t-\tau_{p} / 2\right)}$ & $\phi_{0}$ & $\cos \frac{\phi_{0}}{2}$ & $\cos \phi_{0}$ & $\frac{1}{8} \sin \phi_{0}$ & $\frac{1}{8} \sin 2 \phi_{0}$ & $\frac{1}{4} \sin \frac{\phi_{0}}{2}$ & $\frac{1}{4} \sin \phi_{0}$ & $\sin \frac{3 \phi_{0}}{2}$ \\
\hline$\pi \delta(t$ & $\pi$ & 0 & -1 & 0 & 0 & $1 / 4$ & 0 & $-1 / 4$ \\
\hline$G_{0.01} 55$ & $\pi$ & 0.0211 & -0.9709 & 0.0104 & 0.000047 & 0.24996 & 0.00023 & -0.2354 \\
\hline$G_{0.10} \sqrt{55}$ & $\pi$ & 0.2107 & 86 & & 0.0047 & 0.2458 & 0.0233 & 1035 \\
\hline$S_{1}$ & $\pi$ & 0 & -0.6135 & 0.0 & 93 & 82 & -0.0737 & -0.1020 \\
\hline$S_{2} 19$ & $\pi$ & 0 & -0.6675 & 0.0250 & 0.0298 & .2414 & -0.0557853 & -0.1171 \\
\hline$Q_{1}$ & $\pi$ & 0 & -0.6761 & 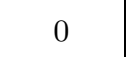 & -0.0079 & .2399 & 0.0027 & -0.1234 \\
\hline$Q_{2} 19$ & $\pi$ & 0 & -0.7138 & 0 & -0.0065 & 2422 & 0.0 & 1342 \\
\hline$W_{11}(\pi)$ & $\pi$ & 0 & 0 & 0.0511 & -0.0039 & 884 & -0 & 0353 \\
\hline$W_{12}(\pi)$ & $\pi$ & 0 & 0 & .0400 & -0.0164 & 1904 & & .0413 \\
\hline & $\pi$ & ( & 0 & & 0.0 & 0072 & & -0.0093 \\
\hline $2(\pi)$ & $\pi$ & 0 & 0 & 0 & 0107 & 0634 & 0.0415 & -0.0035 \\
\hline (- & $\pi$ & 0 & 0 & ( & 61 & 36 & & 14 \\
\hline И & $\pi$ & c & c & ( & & & & \\
\hline$F_{1}$ & $\pi$ & 0.0018 & 0.3307 & 0.0237 & -0.01018 & 1134 & -0 . & 0.0680 \\
\hline $\operatorname{PKRU}_{1}(\pi)[54$ & $\pi$ & 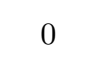 & -0.6880 & 0.0278 & 0.0368 & 0.2420 & -0.0614 & -0.1250 \\
\hline $\operatorname{PKRU}_{2}(\pi)[54$ & $\pi$ & 0 & -0.1501 & 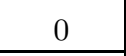 & -0.0078 & 0 & 0.0866 & -0.0047 \\
\hline$\frac{\pi}{2} \delta\left(t-\tau_{p} / 2\right)$ & $\pi / 2$ & $\sqrt{2} / 2$ & - & & 0 & $\sqrt{2} / 8$ & $\overline{1 /}$ & $\sqrt{2} / 8$ \\
\hline$(\pi / 2)$ & $\pi / 2$ & 0.7136 & 0.02 & 0.1 & 0 & 67 & 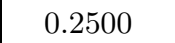 & 0.1872 \\
\hline /2) & $/ 2$ & 0.7722 & 0.210 & 0.1388 & 0.0872 & 0.1706 & & 0.2599 \\
\hline$Q_{1}(\pi / 2) 52$ & $\pi / 2$ & 0 & -0.2906 & 0 & -0.0023 & 0.2021 & 0.0040 & -0.0508 \\
\hline$Q_{2}(\pi / 2) 52$ & $\pi / 2$ & 0 & -0.0109 & 0 & 0.0035 & 0.1617 & 30 & 0.00077 \\
\hline $1(\pi / 2)$ & $\pi / 2$ & 0 & 0 & 010 & -0.0022 & 0.1787 & & .0193 \\
\hline & $\pi / 2$ & c & 0 & -0.0 & -0 & .1756 & & 103 \\
\hline & $\pi$ & ( & 0 & 0 & & 1796 & & 190 \\
\hline$W_{22}(\pi / 2)$ & $\pi / 2$ & 0 & . & - & -0.0021 & 0.1771 & 0.0324 & 0.0129 \\
\hline $\operatorname{PKRU}_{1}(\pi / 2) 54$ & $\pi / 2$ & 0 & -0.2529 & 0.02 & -0.0242 & 0.1992 & 0.0541 & -0.0422 \\
\hline $\operatorname{KRU}_{2}(\pi / 2) 54$ & $|\pi / 2|$ & 0 & 0.2711 & 0 & 0.0127 & 0 & 0.0483 & 0.00028 \\
\hline
\end{tabular}

TABLE I: Expansion coefficients for different pulse shapes. See text for the definitions.

pulses. Specifically,

$$
\begin{aligned}
& \bar{\Gamma}^{(0)}(\mathbf{1 6 a})=\bar{\Gamma}^{(0)}(\mathbf{8} \mathbf{s})=\left.\bar{\Gamma}^{(0)}(\mathbf{8} \mathbf{a})\right|_{v \rightarrow 0} \\
& \bar{\Gamma}^{(1)}(\mathbf{1 6 a})=\bar{\Gamma}^{(1)}(\mathbf{8} \mathbf{a})=0
\end{aligned}
$$

where $\bar{\Gamma}^{(0)}(\mathbf{8} \mathbf{a})=\bar{\Gamma}^{(0)}(\mathbf{4} \mathbf{p})$ is given by Eq. (55). We should mention that for Hamiltonian evolution, $\hat{\gamma}=0$, one could achieve $\bar{\Gamma}^{(0)}(\mathbf{8} \mathbf{s})=\bar{\Gamma}^{(1)}(\mathbf{8} \mathbf{s})=0$ by using second-order pulses with $v=\alpha=0$ [35, 52].

Comparing these results with the analogous sequences of hard pulses, we see that an equivalent cancellation with soft pulses requires careful pulse shaping or doubling the number of pulses in the sequence. On the other hand, while sequences of hard $\pi$ pulses do not modify the diagonal part of the effective decoherence operator, this is not so with shaped pulses where $v_{2} \neq 0$. This corresponds to a reduction of the effective decoherence rate $\bar{T}_{1}^{-1}$ due to redistribution between the channels. For example, with the NMR decoherence model, Eq. (17), the inverse decoherence time becomes

$$
\bar{T}_{1}^{-1}=2 \gamma-\left(\gamma-\gamma_{\phi}\right) \frac{1+v_{2}}{2}
$$

for $\gamma>\gamma_{\phi}$ and limited control fields $\left(\left|v_{2}\right|<1\right)$, this is smaller than the original $T_{1}^{-1}=2 \gamma$. Note that the reduction is achieved solely by redistribution of decoherence between the channels, that is, by a corresponding increase in $\bar{T}_{2}^{-1}$. For full symmetrization [Eq. (28)] one needs $v_{2}=1 / 3$.

\section{Sequences of finite-length $\pi / 2$ pulses}

For the family of sequences $\mathbf{1 2}, \mathbf{2 4}, \mathbf{4 8}$ [see Eqs. (35) (37)] of $\pi / 2$ pulses, the results are similar to those with hard pulses. Specifically, when used with NMR decoherence model (17), the sequence $\mathbf{1 2}$ achieves leading-order coherence matrix symmetrization but not the decoupling. The symmetrized (and phase ramped) sequences $\mathbf{2 4}$ and 48 achieve symmetrization and decoupling in the leading 
and subleading orders, respectively. More explicitly, for symmetric pulse shapes and the decoherence model (17),

$$
\bar{\Gamma}^{(0)}(\mathbf{2 4})=\bar{\Gamma}^{(0)}(\mathbf{4 8})=\frac{2}{3}\left(2 \gamma+\gamma_{\phi}\right) \mathbb{1}, \bar{\Gamma}^{(1)}(\mathbf{4 8})=0
$$

The non-zero matrix elements in $\bar{\Gamma}^{(1)}(\mathbf{2 4})$ contain terms scaling as the products of $\left(\gamma-\gamma_{\phi}\right)$ and the external field components $B_{\mu}$ in all combinations; all of the coefficients cannot be eliminated merely by pulse shaping. In the special case $B_{\mu}=0$, the correction $\bar{\Gamma}^{(1)}(\mathbf{2 4}) \propto\left(\gamma-\gamma_{\phi}\right)^{2}$ is suppressed for pulse shapes with $v_{2}=\alpha_{2}=0$.

With generic decoherence matrix $\hat{\gamma}, \bar{\Gamma}^{(1)}(48)=0$ for 90-degree pulses with $v=v_{2}=0$. In this case the full symmetrization of the decoherence matrix can be achieved as long as $\gamma_{x x}=\gamma_{y y}$, which can be always made to be the case by an appropriate choice of the basis in the $x-y$ plane.

\section{Pulse shaping}

The obtained analytical results imply two possible applications for pulse shaping. First, a pulse can be shaped to have the coefficients $v, \alpha, \alpha_{2}$, and $\zeta_{2}$ zero, so that the second-order correction to effective decoherence operator is zero already for the 8-pulse sequence $\mathbf{8 s}$. The resulting effective decoherence operator $\bar{\Gamma}$ will be diagonal, with the matrix elements determined by the diagonal terms of the original decoherence matrix $\hat{\gamma}$ and the parameter $v_{2}$ [cf. the diagonal elements in Eq. (55)]. We note that up to terms of second order in pulse duration, one might as well use the longer sequence $\mathbf{1 6 a}$ to cancel the terms in $\bar{\Gamma}$ proportional to coefficients $v, \alpha, \alpha_{2}, \zeta_{2}$.

The value $v_{2}=-1$ corresponds to an ideal hard inversion pulse, in which case the diagonal matrix elements of the matrix $\bar{\Gamma}$ are not modified. On the other hand, for $v_{2}=1 / 3$, assuming $\gamma_{x x}=\gamma_{y y}$ (which can always made to be the case by a rotation in the $x-y$ plane), the effective decoherence operator is fully symetrized [see Eq. (28)]. This is the second potential application for pulse shaping. Again, to second order, the same symmetrization can be also achieved with the composite-pulse sequence 48 using $\pi / 2$-pulses with $v=v_{2}=0$ (generic decoherence matrix), or any pulses for the special case of NMR decoherence model (17).

We followed Refs. 19, 52 to construct a number of new $\pi / 2$ and inversion $\left(\phi_{0}=\pi\right)$ pulse shapes with $v=v_{2}=0$ $\left[W_{1 s}\left(\phi_{0}\right)\right], v=v_{2}=\alpha=0\left[W_{2 s}\left(\phi_{0}\right)\right]$, and $v=v_{2}=$ $\alpha=\zeta_{2}=0\left[W_{3 s}\left(\phi_{0}\right)\right]$, where the parameter $s=1,2$ determines the number of derivatives that vanish at the ends of the pulse interval (2 and 4 , respectively). The parameters of the new and previously constructed pulses are listed in Tab. If, and their coefficients in Tab. II. The shapes of the pulses we used in the simulations are shown in Fig. 2.

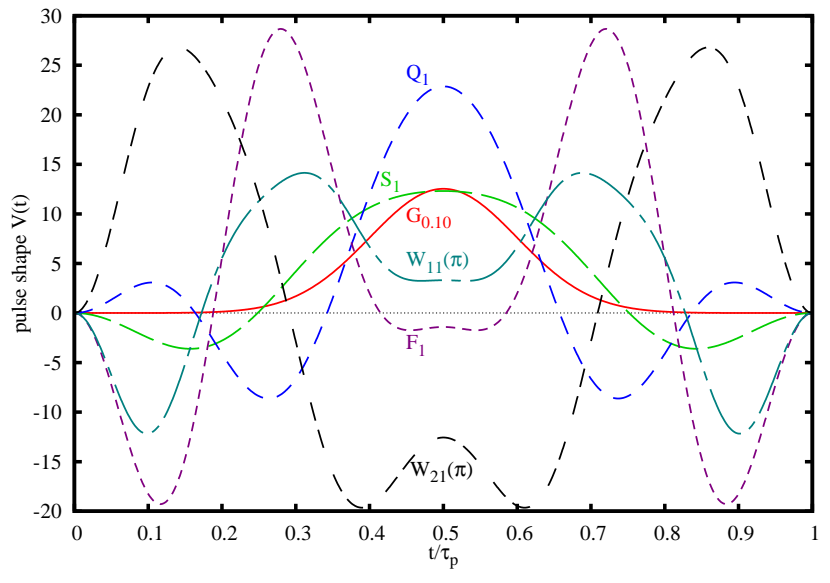

FIG. 2: (Color online) Pulse shapes used in the simulations. The Gaussian shape $G_{x}(y)=\left[(2 \pi)^{1 / 2} x \tau_{p}\right]^{-1} \exp \left(-0.5 y^{2} / x^{2}\right)$ with $x=0.10$ and $y=t / \tau_{p}-0.5$ is $\sqrt{2}$ wider than that used in Ref. 35. The shapes $S_{1}, Q_{1}$ were originally constructed in Ref. 19]. The shapes $F_{1}, W_{11}, W_{21}$ are constructed in this work, see Tab. II for the coefficients.

\section{SIMULATIONS}

Our analytical analysis was only done to second order cumulant expansion, and assuming time-independent fields $B_{\mu}$. The results are asymptotically true in the limit where the sequences are sufficiently short, that is, $\tau B \ll 1, \gamma \tau \ll 1, \tau \ll \tau_{c}$, where $\tau_{c} \sim \omega_{c}^{-1}$ is the correlation time of the slow part of the bath. To check for possible effect of ignored terms, we performed numerical simulations of the controlled dynamics of a qubit in the presence of time-dependent correlated classical Gaussian fields $B_{\mu} \equiv B_{\mu}(t)$, as well as Markovian decoherence described by the Lindblad equation (11), $(12)$. We specifically used the NMR decoherence model (17) in the regime dominated by phase fluctuations, $\gamma_{\phi}>0, \gamma=0$ [note that with a non-zero $\gamma$ the decoherence matrix can be decomposed as $\hat{\gamma}=\gamma \mathbb{1}+\left(\gamma_{\phi}-\gamma\right) \operatorname{diag}(0,0,1)$; the part proportional to the identity matrix commutes with decoupling pulses and can be trivially eliminated.] The fields $B_{\mu}(t)$ were chosen as zero-average correlated Gaussian random functions with the correlation function

$$
\left\langle B_{\mu}(t) B_{\nu}\left(t^{\prime}\right)\right\rangle=\delta_{\mu \nu} B_{0}^{2} g\left(t-t^{\prime}\right), \quad g(t)=e^{-t^{2} / 2 \tau_{c}^{2}},
$$

and the fixed value of the correlation time $\tau_{c}=8 \tau_{p}$. The control fields $V_{\mu}(t), \mu=x, y$, were generated according to the chosen decoupling sequence and the pulse shape, with the $k$-th pulse of the sequence fitting in the interval $(k-$ 1) $\tau_{p} \leq t \leq k \tau_{p}$; the corresponding decoupling period $\tau=$ $n \tau_{p}$ is given by the number $n$ of pulses in the sequence. For every sequence, pulse shape, and the realization of the random fields $B_{\mu}(t)$, we solved Eqs. (15), (16) with $\overrightarrow{\mathcal{R}}=0$ for three sets of initial conditions with $R_{\mu}=1$, $\mu=x, y, z$; the solutions correspond to the columns of the evolution matrix $\hat{Q}(t)$ [Eq. (21)]. The average decoupling 


\begin{tabular}{c|c|c|c|c|c|c|c|c} 
pulse & $\mathrm{A} 0$ & $\mathrm{~A} 1$ & $\mathrm{~A} 2$ & $\mathrm{~A} 3$ & $\mathrm{~A} 4$ & $\mathrm{~A} 5$ & $\mathrm{~A} 6$ & $\mathrm{~A} 7$ \\
\hline$F_{1}$ & 0.5 & -1.419474 & -2.048028 & 1.549555 & 1.435813 & -0.017867 & & \\
$W_{11}(\pi)$ & 0.5 & -1.242022 & -1.009075 & 0.700828 & 0.530624 & 1652161644 & 0.277982 & 0.241663 \\
$W_{12}(\pi)$ & 0.5 & -1.291342 & -0.753726 & 1.499438 & 0.364546 & 0.012680 & -0.069983 & -0.261614 \\
$W_{21}(\pi)$ & 0.5 & 3.056086 & -1.295369 & -1.689687 & -0.062202 & -0.366646 & -0.142183 & \\
$W_{22}(\pi)$ & 0.5 & 2.776007 & -2.473314 & -1.782314 & 0.958211 & -0.444991 & 0.300165 & 0.166236 \\
$W_{31}(\pi)$ & 0.5 & -1.110710 & -3.692547 & 1.248118 & 0.990698 & 1.394824 & 0.669618 & \\
$W_{32}(\pi)$ & 0.5 & -1.686664 & -2.108402 & 3.362253 & 1.029286 & -0.260405 & -0.836068 & \\
\hline$W_{11}(\pi / 2)$ & 0.25 & 2.011311 & 0.041292 & 1.381531 & 0.262448 & 0.076040 & & \\
$W_{12}(\pi / 2)$ & 0.25 & 2.023581 & 0.920572 & 1.341484 & -0.113434 & -0.144034 & -0.231008 & \\
$W_{21}(\pi / 2)$ & 0.25 & 2.018463 & 0.588295 & 1.393403 & -0.206226 & 0.095943 & -0.1029524 & \\
$W_{22}(\pi / 2)$ & 0.25 & 2.018283 & 0.608538 & 1.386685 & 0.088935 & 0.024615 & -0.134584 & -0.205904
\end{tabular}

TABLE II: Pulse coefficients for various shapes constructed for this work. The control field during the pulse, $0<t<\tau_{p}$, is represented as $V(t)=2 \pi \sum_{n} A_{n} \cos \left(2 \pi n t / \tau_{p}\right)$.

fidelity (29) was then evaluated using the expression

$$
\left\langle F\left(t_{s}\right)\right\rangle=\frac{1}{2}\left(1+\frac{\operatorname{Tr} \hat{Q}\left(t_{s}\right)}{3}\right) .
$$

at the time moments $t_{s}$ commensurate with the sequence duration $t_{s}=s \tau=s n \tau_{p}$.

The calculation results for a particular sample of the fields $B_{\mu}(t)$ and several sequences of inversion $\left(\phi_{0}=\pi\right)$ Gaussian pulses are shown in Fig. 3. The line marked as "ideal" corresponds to the best achievable fidelity

$$
\langle F(t)\rangle_{\text {ideal }}=\frac{1}{3}\left(2+e^{-\gamma_{\phi} t}\right)
$$

with the chosen value $\gamma_{\phi}=2 \pi \times 10^{-3} / \tau_{p}$ at the end of the simulation we get $\left\langle F\left(512 \tau_{p}\right)\right\rangle_{\text {ideal }} \approx 0.680$. The points marked "no control" corresponds to average fidelity in the absence of decoupling pulses; the coherence is lost after just a few pulse durations. While decoupling is not particularly efficient (and noise is evident) for the 4-pulse sequence (set of points marked $\mathbf{4 p}$ in Fig. 3), the fidelity is improved and noise markedly reduced for the sequence $\mathbf{8 s}$, and even more so for the sequence 16a. In fact, the points for sequence $\mathbf{1 6 a}$ are very close to the dashed line which represents the leading-order contribution at $B=0$, see Eq. (63) below.

In the presence of the Markovian decoherence, we can view the average infidelity as composed of three terms. First is the inavoidable infidelity due to the Markovian decoherence alone, in the absence of any control fields or low-frequency noise. For the case of pure dephasing, it is given by $1-\langle F(t)\rangle_{\text {ideal }}$, see Eq. (62). For the value of $\gamma_{\phi}$ used in our simulation, $\langle F(t)\rangle_{\text {ideal }}$ is shown in Fig. 3 with the solid line. Second is the fidelity loss due to the redistribution of the original decoherence rate(s) over the channels. In our simulations, for the sequences $\mathbf{4 p}, \mathbf{8 s}$, 16a of $\pi$-pulses other than $G_{0.10}$, this part of the average infidelity is to a very good accuracy determined by the coefficient $v_{2}$ of the corresponding pulses,

$$
\langle F(t)\rangle_{0}=\frac{1}{6}\left(3+e^{-\gamma_{1} \text { eff } t}+2 e^{-\gamma_{2} \text { eff } t}\right),
$$

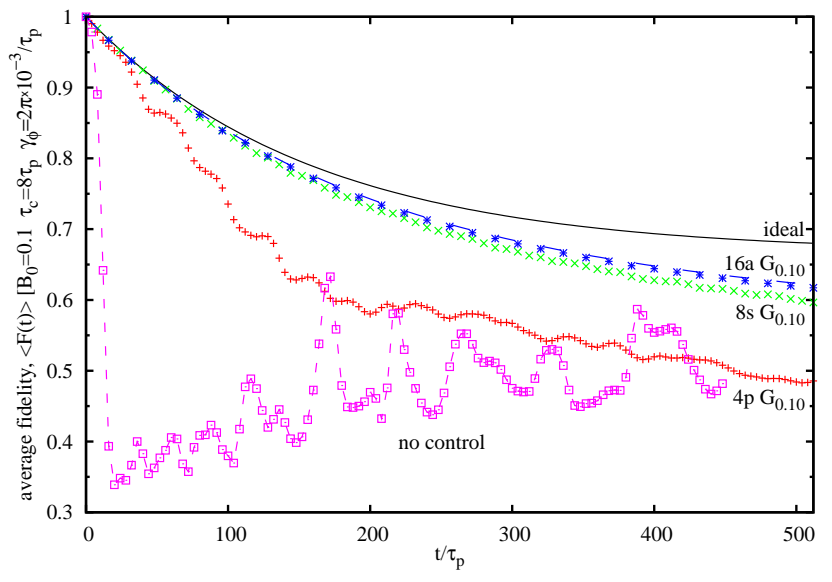

FIG. 3: (Color online) Single-qubit average fidelity for a particular realization of time-dependent fields and Markovian dephasing with the rate $\gamma_{\phi}=2 \pi \times 10^{-3} / \tau_{p}$. Open boxes connected with the dashed line marked "no control" correspond to the absence of decoupling. Other symbols are numerical data for the indicated decoupling sequences of Gaussian inversion pulses. The black solid line marked "ideal" corresponds to maximum achievable average fidelity. Dashed line corresponds to Eq. 63 with $v_{2}=-0.7086$ as appropriate for the

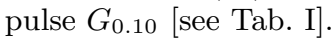

$$
\gamma_{1 \mathrm{eff}}=\gamma_{\phi} \frac{1+v_{2}}{2}, \gamma_{2 \mathrm{eff}}=\gamma_{\phi} \frac{3-v_{2}}{4},
$$

see Tab. 目and Fig. 1 .

Finally, the third part of the average infidelity is entirely due to the presence of the low-frequency random fields; it is defined as the difference between the average fidelity of the controlled system in the absence of low-frequency fields, and that in their presence, $\Delta F(t) \equiv$ $\langle F(t)\rangle_{0}-\langle F(t)\rangle$. It is this quantity that directly characterizes the ineffectiveness of the decoupling against the low-frequency fields.

The corresponding plots are shown in Figs. 5 (sequence 8s) and 6 (sequence 16a), as well as in Figs. 8 , 8 with 


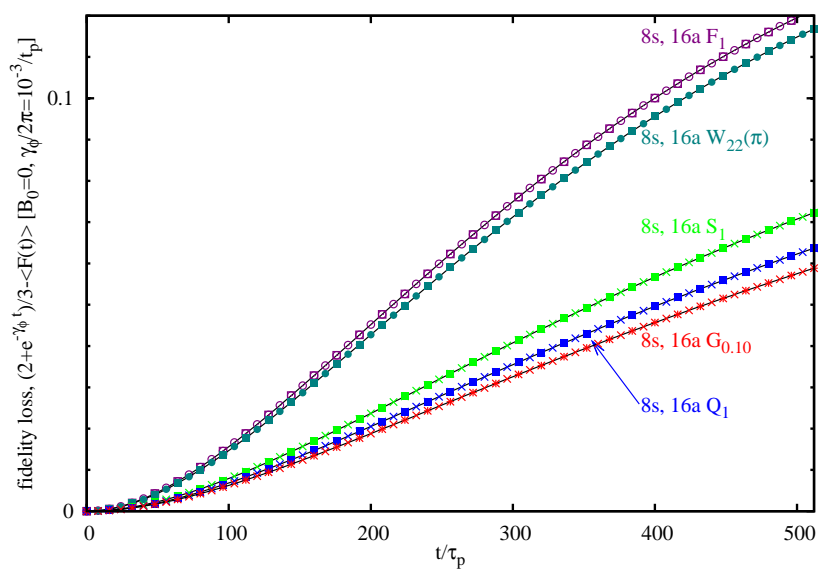

FIG. 4: (Color online) Single-qubit average fidelity loss due to redistribution of the decoherence rates over directions in the absence of low-frequency fields. Symbols are numerical data for pulses and sequences as indicated, and lines are computed analytically using Eq. 63) with corresponding $v_{2}$ from Tab. If This contribution to infidelity would be zero for hard ( $\delta$-function) pulses with $v_{2}=-1$.

$\gamma_{\phi}=0$ for comparison purposes. The general trend is consistent with the expectations based on analytical expansion. For example, with the Gaussian pulses we get the largest error, with or without the Markovian dephasing; the decoupling error is smaller with the $\mathbf{1 6 a}$ sequence [as compared with the $\mathbf{8 s}$ ] where the corrections including the subleading order are suppressed. Similarly, specially designed pulse shapes with a larger number of suppressed parameters lead to improved performance in the presence of Markovian dephasing: compare, e.g., the curves for pulses $S_{1}, W_{11}(\pi)$, and $W_{21}(\pi)$.

One marked exception is the pulse shape $F_{1}$, for which $v_{2} \approx+1 / 3$ while the other coefficients have magnitude comparable to those for, e.g., the shape $S_{1}$ (apart from the coefficient $v$ which is zero for the shape $S_{1}$ ). While in the absence of Markovian dephasing, the decoupling error for the pulse $F_{1}$ is qualitatively similar to that for the 1st-order shapes [Figs. 7, 8], with $\gamma_{\phi} \neq 0$ the decoupling error is actually closer to that for the second-order pulses with the sequence $\mathbf{8 s}$ [Fig. 5] and is the smallest of the shapes we considered for the sequence 16a [Fig. 6]. We believe that this, at least in part, is associated with the symmetrization of the effective decoherence tensor achieved in the presence of the pulse $F_{1}$, which renders the resulting Markovian decoherence (effectively, the amplitude damping) independent from the decoupling, and thus reduces the contribution from high orders not included in our analytical calculations.

In comparison with the sequences of $\pi$-pulses, the basic unit in the sequences of $\pi / 2$ pulses, 12p, 24s, and $\mathbf{4 8} \mathrm{s}$, is three times longer. As a result, weaker random fields $B_{\mu}(t)$ with longer correlation times are required to achieve comparable decoupling accuracy. In addition, with longer coherence time, longer evolution times are

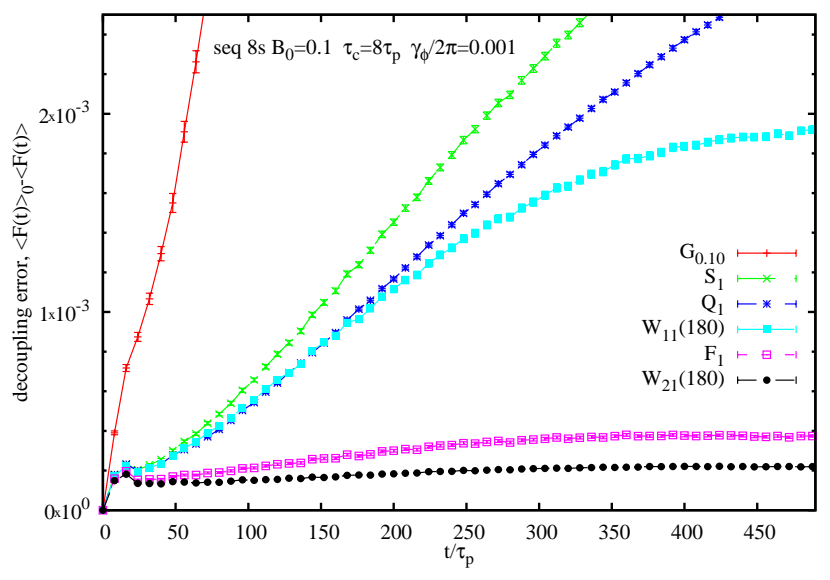

FIG. 5: (Color online) Single-qubit decoupling error for the 8pulse sequence $8 \mathrm{~s}$ with several pulse shapes, as indicated. The decoupling error is small for the second-order pulse $W_{21}(\pi)$ and, somewhat surprisingly, for the symmetrizing shape $F_{1}$. With $\gamma_{\phi}=0$, the infidelity for this pulse shape with sequence $\mathbf{8}$ grows similarly to that of first-order pulses $S_{1}$ and $W_{11}$ [Fig. 7].

required to separate the effects of the transients near the beginning of the simulation interval. By these reasons, we do not discuss the performance of these sequences in detail. For the parameters as in Figs. 5, 6, the decoupling error with the sequences $\mathbf{2 4}$ s and $48 \mathrm{~s}$ saturates at around twenty times that for the sequences $\mathbf{8 s}, \mathbf{1 6 a}$, while with $\tau_{c}=32 \tau_{p}$ and the same $B_{0}=0.1 / \tau_{p}$, the decoupling error is comparable to that in Figs. 5, 6. Note that these decoupling errors are still small compared to the fidelity loss due to redistribution of the decoherence rates over directions, which for these sequences is identical to that of sequences $\mathbf{8 s}, \mathbf{1 6} \mathbf{a}$ with the symmetrizing pulse $F_{1}$ [see Fig. 4.

\section{CONCLUSIONS}

We considered the dynamical decoupling in a simple decoherence model simulating the presence of both lowand high-frequency environment modes [Fig. 1], having in mind applications of DD in combination with QECCs. We modeled the effect of low-frequency degrees of freedom in terms of classical correlated noise, and that of the fast degrees of freedom with the help of the Markovian master equation in the Lindblad form, see Eqs. (11), (10). The combined effect is the non-Hermitian evolution (15) of the Bloch vector with the instantaneous decoherence operator (16) [see Eq. (17) for the special case of NMR decoherence model]. In the presence of dynamical decoupling with the sequence period $\tau$, the values of the average qubit fidelity at the commensurate time moments $\tau_{s}=s \tau$ are determined by the effective decoherence matrix $\bar{\Gamma}$ [Eq. (23)], an analogue of the average Hamiltonian. The trace of the effective decoherence ma- 


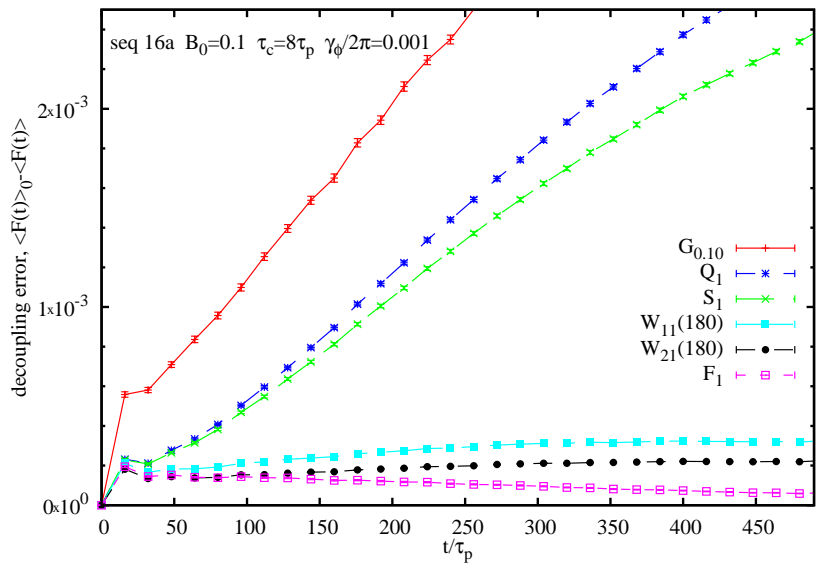

FIG. 6: (Color online) As in Fig. 5 but for 16-pulse sequence 16a. For this sequence, and with time-independent fields $B_{\mu}$, there would be no contribution from the first two orders of the effective decoherence operator [Eqs. (56), (57)]; the decoupling error is determined by the non-adiabaticity and by the terms of higher order. Generic pulse shapes $G_{0.10}, S_{1}$, and $Q_{1}$ show the largest decoupling error, while it is the smallest for the symmetrizing shape $F_{1}$ (technically, zeroth order, $v \neq 0$; $\left.v_{2} \approx 1 / 3\right)$. For shapes $F_{1}, W_{11}$, and $W_{21}$, the decoupling error here is actually smaller than the infidelity in Fig. 8. We believe this is an artefact of the definition of the decoupling error related to the full average fidelity approaching saturation at $1 / 2$ [Fig. 3].

trix is conserved, see Eq. (27); in agreement with general expectations, this implies that dynamical decoupling can only decrease the fidelity in the presence of Markovian degrees of freedom alone.

This is always the case for DD with soft pulses, which necessarily leads to redistribution of the decoherence rates over the directions, leading to some fidelity reduction even in the absence of the slow degrees of freedom. For evolution time small compared to the decoherence time $\sim \gamma^{-1}$ (as required for efficient error correction), this reduction is a relatively small effect. On the flip side, this redistribution causes symmetrization of decoherence operator which reduces the effect of the non-Hamiltonian dynamics associated with the fast degrees of freedom on the decoupling accuracy. For example, if the symmetrization (28) is achieved at the end of a basic decoupling cycle, decoherence is expected to have no effect on additional cancellations achieved in a supercycle obtained by, e.g., phase-ramping the basic cycle.

To analyze relative importance of these effects, we considered several decoupling sequences of both hard and soft (generic 1st- and 2nd-order and specially shaped) pulses which lead to various degrees of symmetrization of the decoherence operator. For such sequences, in the static limit where the slow degrees of freedom become time-independent, we constructed analytically the first two terms of the (cumulant) expansion of the effective decoherence operator $\bar{\Gamma}$ in powers of the sequence pe-

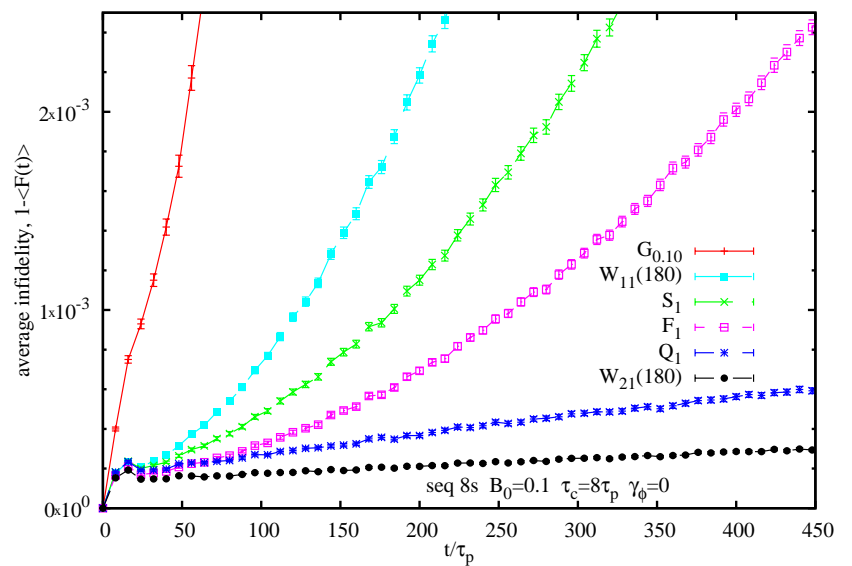

FIG. 7: (Color online) As in Fig. 5 but in the absence of Markovian dephasing, $\gamma_{\phi}=0$. Infidelity curves with 2ndorder pulses $Q_{1}$ and $W_{21}$ have a finite slope, unlike the plots in Ref. 21 where a smaller r.m.s. value $B_{0}$ of the slow fields was used. We checked that the slopes for the 2nd-order pulse shapes are greately reduced if $B_{0}$ is reduced by a factor of two (not shown).

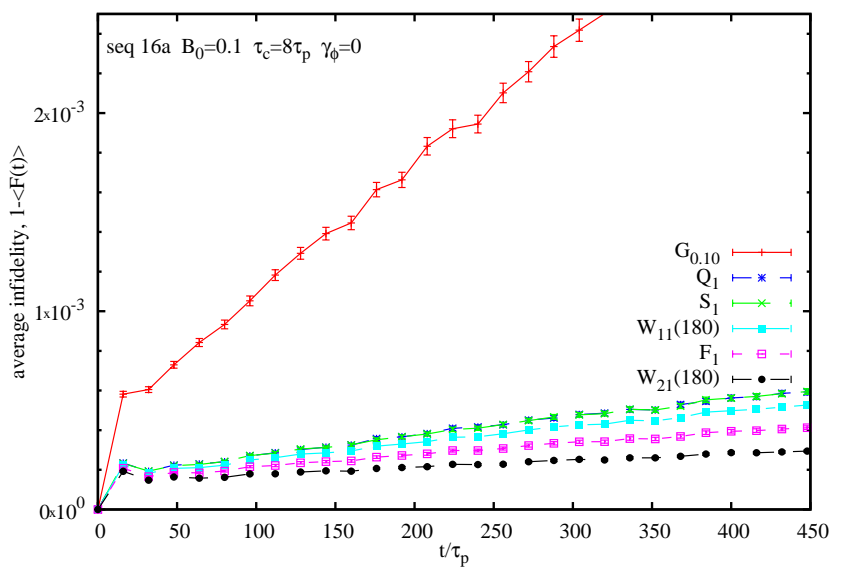

FIG. 8: (Color online) As in Fig. / but for the sequence 16a. The slopes for all the pulse shapes excluding $G_{0.10}$ are greately reduced if $B_{0}$ is reduced by a factor of two (not shown). For the Gaussian pulses $G_{0.10}$, the second-order cancellation is achieved only after the complete sequence with the duration $\tau=16 \tau_{p}$; the slope reduction happens for slower fields $B_{\mu}(t)$ with $\tau_{c}=16 \tau_{p}$ (not shown).

riod $\tau$. With the help of pulse shaping and/or phase ramping, we could ensure that the leading-order average decoherence operator $\bar{\Gamma}^{(0)}$ is diagonal and independent of the components of the slow field, while the subleading contribution disappears identically, $\bar{\Gamma}^{(1)}=0$. Results of Refs. 19, 21 suggest that such sequences might also be effective in the presence of sufficiently weak slowly-varying fields.

Numerical simulations generally confirmed these expectations. In the studied parameter range, the best 
decoupling accuracy is achieved with the sixteen-pulse sequence 16a. With time-independent external fields the sequence provides complete decoupling of the slow fields in the first two orders of the cumulant expansion independent of the shape of symmetric inversion pulses, see Eqs. (56), (57). The effects of higher order terms and non-adiabaticity of the fields $B_{\mu}(t)$ are captured in the simulations, see Sec. IV. The best performance is achieved with the "symmetrizing" pulse shape $F_{1}$ constructed with the only requirement that the diagonal part of the leading-order effective decoherence matrix (55) for the 4 -pulse sequence $\mathbf{4} \mathbf{p}$ is symmetric, i.e., $v_{2} \approx+1 / 3$, see Sec. III B 1 and Tab. I In fact, in our simulations the part of the coherence loss associated with the slow fields is smaller than that in the absence of the high-frequency degrees of freedom, cf. Figs. 5, 6 and 8, 8. Overall, the suppression of the decoherence due to slow fields associated with the dynamical decoupling increases the coher- ence time by orders of magnitude.

We thus demonstrated in principle the effectiveness of DD in suppressing the effect of low-frequency degrees of freedom in the presence of high-frequency modes which cannot be eliminated by dynamical decoupling. This opens up possible applications in combined coherence protection techniques concatenating dynamical decoupling with QECC at higher levels. We postpone the corresponding discussion to a futher publication 56.

\section{Acknowledgments}

We are grateful to Sasha Korotkov, Daniel Lidar, Len Mueller, and Götz Uhrig for useful discussions. This research was supported in part by the NSF grant No. 0622242 .
[1] C. P. Slichter, Principles of Magnetic Resonance, Springer-Verlag, New York, 3rd edition, 1992.

[2] P. Hodgkinson and L. Emsley, Prog. NMR Spectr. 36, 201 (2000).

[3] L. M. K. Vandersypen and I. L. Chuang, Reviews of Modern Physics 76, 1037 (2004).

[4] U. Haeberlen and J. S. Waugh, Phys. Rev. 175, 453 (1968).

[5] U. Haeberlen, J. S. Waugh, Phys. Rev. 185, 420 (1969).

[6] A. Pines, J. S. Waugh, J. Mag. Res. 8, 354 (1972).

[7] A. Pines, J. S. Waugh, Phys. Lett. A 47, 337 (1974).

[8] L. Viola and S. Lloyd, Phys. Rev. A 58, 2733 (1998).

[9] D. Vitali and P. Tombesi, Phys. Rev. A 59, 4178 (1999).

[10] L. Viola, E. Knill, and S. Lloyd, Phys. Rev. Lett. 82, 2417 (1999).

[11] L. Viola, S. Lloyd, and E. Knill, Phys. Rev. Lett. 83, 4888 (1999).

[12] L. Viola, E. Knill, and S. Lloyd, Phys. Rev. Lett. 85, 3520 (2000).

[13] D. Vitali and P. Tombesi, Phys. Rev. A 65, 012305 (2002).

[14] C. Uchiyama and M. Aihara, Phys. Rev. A 66, 032313 (2002).

[15] M. S. Byrd and D. A. Lidar, Phys. Rev. A 67, 012324 (2003).

[16] K. Shiokawa and D. A. Lidar, Phys. Rev. A 69, 030302(R) (2004).

[17] L. Faoro and L. Viola, Phys. Rev. Lett. 92, 117905 (2004).

[18] P. Facchi et al., Phys. Rev. A 71, 022302 (2005).

[19] P. Sengupta and L. P. Pryadko, Phys. Rev. Lett. 95, 037202 (2005).

[20] K. Khodjasteh and D. A. Lidar, Phys. Rev. Lett. 95, 180501 (2005).

[21] L. P. Pryadko and P. Sengupta, Phys. Rev. B 73, 085321 (2006).

[22] P. Chen, Phys. Rev. A 75, 062301 (2007).

[23] G. S. Uhrig, Phys. Rev. Lett. 98, 100504 (2007).

[24] T. E. Hodgson, L. Viola, and I. D'Amico, Phys. Rev. B 78, 165311 (2008).
[25] S. Pasini, T. Fischer, P. Karbach, and G. S. Uhrig, Phys. Rev. A 77, 032315 (2008).

[26] M. S. Byrd and D. A. Lidar, J. of Modern Opt. 50, 1285 (2003).

[27] L. Viola, Phys. Rev. A 66, 012307 (2002).

[28] M. Möttönen, R. de Sousa, J. Zhang, and K. B. Whaley, Phys. Rev. A 73, 022332 (2006).

[29] P. Kuopanportti et al., Phys. Rev. A 77, 032334 (2008).

[30] L. Cywiński, R. M. Lutchyn, C. P. Nave, and S. D. Sarma, Phys. Rev. B 77, 174509 (2008).

[31] P. Rebentrost, I. Serban, T. Schulte-Herbrüggen, and F. K. Wilhelm, Phys. Rev. Lett. 102, 090401 (2009).

[32] G. S. Uhrig, New J. Phys. 10, 083024 (2008).

[33] J. S. Waugh, L. M. Huber, and U. Haeberlen, Phys. Rev. Lett. 20, 180 (1968).

[34] J. S. Waugh, C. H. Wang, L. M. Huber, and R. L. Vold, J. Chem. Phys. 48, 652 (1968).

[35] L. P. Pryadko and G. Quiroz, Phys. Rev. A 77, 012330/1 (2007).

[36] O. V. Konstantinov and V. I. Perel, Zh. Exp. Teor. Fiz. 39, 197 (1960).

[37] E. B. Davies, Comm. Math. Phys. 39, 91 (1974).

[38] M. I. Dykman, Physica Status Solidi (b) 88, 463 (1978).

[39] R. Alicki, Phys. Rev. A 40, 4077 (1989).

[40] A. G. Kofman and G. Kurizki, Phys. Rev. Lett. 93, 130406 (2004).

[41] G. Lindblad, Commun. Math. Phys. (1976).

[42] R. Alicki, D. A. Lidar, and P. Zanardi, Phys. Rev. A 73, 052311 (2006).

[43] I. Gourlay and J. F. Snowdon, Phys. Rev. A 62, 022308 (2000).

[44] L. Ioffe and M. Mézard, Phys. Rev. A 75, 032345 (2007).

[45] Z. W. E. Evans, A. M. Stephens, J. H. Cole, and L. C. L. Hollenberg, unpublished, preprint arXiv:0709.3875 (2007).

[46] P. K. Sarvepalli, A. Klappenecker, and M. Rötteler, Proc. R. Soc. A 465, 1645 (2009).

[47] P. Aliferis and J. Preskill, Phys. Rev. A 78, 052331 (2008).

[48] A. M. Stephens, Z. W. E. Evans, S. J. Devitt, , and 
L. C. L. Hollenberg, Phys. Rev. A 77, 062335 (2008).

[49] P. Zanardi, Phys. Lett. A 258, 77 (1999).

[50] W.-K. Rhim, D. D. Elleman, and R. W. Vaughan, J. Chem. Phys. 59, 3740 (1973).

[51] G. A. Morris and A. Gibbs, Magnetic Resonance in Chemistry 29, 83 (1991).

[52] L. P. Pryadko and P. Sengupta, Phys. Rev. A 78, 032336 (2008).
53] W. S. Warren, J. Chem. Phys. 81, 5437 (1984).

[54] S. Pasini, P. Karbach, C. Raas, and G. S. Uhrig, unpublished, preprint arXiv:0906.2070 (2009).

[55] C. Bauer, R. Freeman, T. Frenkiel, J. Keeler, and A. J. Shaka, J. Mag. Res. 58, 442 (1984).

[56] Y. Li, D. Lidar, and L. P. Pryadko, In preparation. 